\title{
Força muscular respiratória e qualidade de vida em crianças e adolescentes com fibrose cística
}

\author{
Respiratory muscle strength and quality of life in children and adolescent with cystic fibrosis
}

Fuerza muscular respiratoria y calidad de vida en niños y adolescentes con fibrosis quística

Francieli Camila Mucha', Suellen Bitencourt Rosa², Ana Carolina da Silva Almeida ${ }^{3}$, Tatiana Godoy Bobbio $^{4}$, Juliana Cardoso ${ }^{5}$, Camila Isabel Santos Schivinski ${ }^{6}$

RESUMO I A fibrose cística (FC) é uma doença genética multissistêmica caracterizada por obstrução crônica que, associada a outras alterações pulmonares, pode comprometer a força muscular respiratória (FMR) e, em consequência, interferir no desempenho de atividades típicas da infância, alterando a qualidade de vida (QV) dessa população. O objetivo do estudo foi avaliar a relação entre FMR e QV de crianças e adolescentes com FC. Tratase de um estudo transversal, que incluiu pacientes com idades entre 6 e 14 anos, provenientes de um centro de referência no Brasil. Realizou-se avaliação antropométrica e da FMR, esta utilizando as pressões inspiratória (PImáx) e expiratória máximas (PEmáx) por meio da manovacuômetria digital (Globalmed ${ }^{\circledR}$ MVD300). Aplicouse o Cystic Fibrosis Questionnaire (QFC), questionário específico para a avaliar a QV nessa doença, nas versões para crianças (QFC-C) e para os pais ou responsáveis (QFC-R). A gravidade da doença foi classificada segundo o escore de Schwachman Doeurshuk (ESD). Dados sobre a colonização de bactérias e o genótipo da doença foram consultados por meio da análise dos prontuários. Analisouse os dados por meio do software SPSS version 20.0 for Windows. Após o teste Shapiro-Wilk, aplicou-se o teste de correlação de Pearson ou Spearman. Em toda a análise foi adotado nível de significância de 5\%. Participaram do estudo 28 crianças (15 meninos) com média de idade de 10,10 1 1,79 anos, as quais apresentaram FMR próxima ao predito e pontuações do QFC indicando boa QV. O ESD relacionou-se negativamente com domínio digestivo $(p=0,03 ; r h o=-0,400)$. A PEmáx apresentou correlação negativa com domínio corpo do QFC-R ( $p=0,002$; rho=0,426) e com domínio tratamento do QFC-C ( $p=0,01$; rho=-0,453). A PImáx apresentou correlação positiva com os domínios físico $(p=0,03$; rho=0,410), emocional $(p=\leq 0,001$; rho=0,573) e tratamento $(p=\leq 0,01$; rho $=-$ 0,605) do QFC-C. A PImáx também mostrou correlação positiva com o domínio respiratório ( $p=0,01$; rho=0,572) do QFC-R. Em conclusão, identificou-se associação entre domínios da QV e FMR, bem como com o ESD e aspectos nutricionais. Essa amostra apresentou valores de FMR acima do esperado e boa QV.

Descritores | Fibrose Cística; Força Muscular; Qualidade de Vida.

ABSTRACT | Cystic fibrosis (CF) is a multisystemic genetic disease characterized by chronic obstruction that, associated with other pulmonary changes, can compromise respiratory muscle strength (RMS) and, consequently, interfere with the performance of typical childhood activities, changing the quality of life(QOL) of this population. The aim of the study was to evaluate the relationship between RMS and QOL of children and adolescents with CF. This is a cross-sectional study, which included patients without acute pulmonary exacerbation, aged between 6 and 14 years, from a reference center in Brazil. Anthropometric and RMS assessments were performed, using maximum inspiratory (MIP) and expiratory (MEP) pressures using digital manovacuometry (Globalmed ${ }^{\circledR}$ MVD300). The Cystic Fibrosis Questionnaire was applied, a specific questionnaire to assess QOL in this disease, in versions for children (QOL-C) and for parents 
or guardians (QOL-P). The severity of the disease was classified according to the Schwachman Doeurshuk score (ESD). Data on colonization and genotype were consulted through the analysis of medical records. The data was analyzed using the SPSS version 20.0 for Windows software. After the Shapiro-Wilkt test, Pearson's or Spearman's correlation test was applied. Throughout the analysis, a significance level of 5\% was adopted. Twenty-eight children (15 boys) participated in the study, with a mean age of $10.10 \pm 1.79$ years, who had a near-predicted RMS and QOL scores indicating good QOL. The ESD was negatively related to the digestive domain $(p=0.03$; rho=-0.400). MEP showed a negative correlation with the QOL-P body domain ( $p=0.002$; rho=-0.426) and with the $Q O L-C$ treatment domain ( $p=0.01$; rho=-0.453). MIP showed a positive correlation with the physical $(p=0.03$; rho=0.410), emotional $(p=\leq 0.001$; rho=0.573) and treatment $(p=\leq 0.01$; rho=-0.605) domains of the QOL-C. MIP also showed a positive correlation with the respiratory domain ( $p=0.01$; rho=0.572) of the $Q O L-P$. In conclusion, an association was identified between QOL and RMS domains, as well as with ESD and nutritional aspects. This sample showed higher than expected RMS values and good QOL.

Keywords | Cystic Fibrosis; Muscle Strength; Quality of Life.

RESUMEN I La fibrosis quística (FQ) es una enfermedad genética multisistémica caracterizada por una obstrucción crónica que, asociada a otras alteraciones pulmonares, puede comprometer la fuerza de los músculos respiratorios (FMR), lo que en consecuencia interfiere en el desempeño de las actividades típicas de la infancia alterando la calidad de vida (CV) de esta población. El objetivo de este estudio fue evaluar la relación entre la FMR y la CV de niños y adolescentes con FQ. Este es un estudio transversal, en el cual participaron pacientes de entre 6 y 14 años de un centro de referencia en Brasil. Se realizaron evaluaciones antropométricas y de FMR, para esta se utilizó presión inspiratoria máxima (PImáx.) y presión espiratoria máxima (PEmáx.) mediante manovacuometría digital (Globalmed MVD300). Se aplicó el Cuestionario de fibrosis quística (CFC) específico para evaluar la CV en esta enfermedad, en versiones para niños (CFC-N) y para padres o tutores (CFC-T). La clasificación de la gravedad de la enfermedad siguió el puntaje de Schwachman Doeurshuk (ESD). Se consultaron datos sobre la colonización bacteriana y el genotipo de la enfermedad mediante el análisis de historias clínicas. Para el análisis de datos se utilizó el software SPSS versión 20.0 para Windows. Tras la prueba de Shapiro-Wilk, se aplicó la prueba de correlación de Pearson o Spearman. El nivel de significancia que se adoptó fue del 5\%. En el estudio participaron 28 niños (15 varones), con promedio de edad de 10,10 $\pm 1,79$ años, que presentaron puntuaciones de FMR cerca al predicho y el puntaje de CFC que indicaba una buena CV. EI ESD se relacionó negativamente con el dominio digestivo $(p=0,03$; rho=-0,400). La PEmáx. presentó una correlación negativa con el dominio cuerpo de CFC-T ( $p=0,002$; rho=-0,426) y con el dominio de tratamiento de CFC-N ( $p=0,01$; rho=-0,453). La PImáx. mostró una correlación positiva con los dominios físico $(p=0,03$; rho=0,410), emocional ( $p=\leq 0,001$; rho=0,573) y tratamiento $(\mathrm{p}=\leq 0,01 ;$ rho=-0,605) del CFC-N. La PImáx. también presentó una correlación positiva con el dominio respiratorio $(p=0,01$; rho=0,572) del CFC-T. En conclusión, se identificó una asociación entre los dominios CV y FMR, así como el ESD y los aspectos nutricionales. Esta muestra presentó valores de FMR superiores a los esperados y una buena $\mathrm{CV}$.

Palabras clave | Fibrosis Quística; Fuerza Muscular; Calidad de Vida.

\section{INTRODUÇÃO}

A Fibrose cística (FC) é uma doença genética multissistêmica do tipo autossômica recessiva, decorrente de uma mutação no gene Cystic Fibrosis Transmembrane Regulator (CFTR) que compromete a proteína reguladora da condutância trans-membrana, resultando em um desequilíbrio na concentração de cloro e sódio das glândulas exócrinas. Trata-se do distúrbio genético recessivo mais comum e limitante da vida na população caucasiana, sendo caracterizado principalmente por alteração pulmonar obstrutiva crônica, insuficiência pancreática e desnutrição ${ }^{1,2}$. Atualmente, a doença pulmonar obstrutiva crônica é a principal causa de

morbidade ${ }^{3}$. Segundo o registro brasileiro de fibrose cística, no ano de 2017,86\% dos óbitos foram decorrentes de alterações respiratórias ${ }^{4}$.

A obstrução das vias aéreas é causada pela produção excessiva de secreção, propiciando a presença de inflamação crônica e infecções recorrentes. Em consequência, o aumento da resistência das vias aéreas e do trabalho respiratório, a hiperinsuflação pulmonar, o déficit nutricional, a alteração da complacência e o desarranjo do equilíbrio tóraco-abdominal, são alguns fatores que podem determinar alterações na força dos músculos respiratórios $(\mathrm{FMR})^{5,6}$.

Alguns estudos apontam para a perda na pressão inspiratória ${ }^{6,7}$ na FC, atribuindo essa diminuição 
da FMR com o comprometimento da capacidade ventilatória, possibilidade de fadiga, intolerância ao exercício e às atividades de vida diárias (AVD) ${ }^{8,9}$. Essa inatividade física aliada à diminuição progressiva do condicionamento físico inicia um ciclo vicioso, no qual a piora da dispneia associa-se à diminuição da atividade física, e isso acaba refletindo nas AVD e em atividades típicas da infância ${ }^{6}$, o que compromete a qualidade de vida $(\mathrm{QV})^{10}$ dessa população.

Deste modo, a mensuração da QV e monitorização da FMR são relevantes na rotina de avaliação de indivíduos com FC, e imprescindíveis no acompanhamento do desenvolvimento da criança. No entanto, na faixa etária pediátrica, poucos trabalhos são realizados especificamente sobre a associação entre QV e a FMR. Sendo assim, o objetivo deste estudo foi analisar a relação entre a FMR e a QV de crianças e adolescentes com FC, e partiu-se da hipótese que essa relação está presente na população estudada.

\section{METODOLOGIA}

Esta pesquisa consiste em um estudo analítico observacional transversal, que incluiu crianças e adolescentes entre 6 e 14 anos de idade, com diagnóstico de FC confirmado pelo teste do suor e exame genético ${ }^{11}$. Trata-se de uma amostra de conveniência composta por pacientes que são acompanhados no ambulatório de FC do Hospital Infantil Joana de Gusmão, em Florianópolis (SC), Brasil, e por crianças e adolescentes vinculados ao programa de extensão "Brincando de Respirar", da Universidade do Estado de Santa Catarina. O projeto foi aprovado pelo Comitê de Ética em Pesquisa do referido hospital sob o registro CAAE: 36493314.8.00005361, sendo respeitados os princípios de ética na pesquisa com seres humanos presentes na resolução 466/12 do Conselho Nacional de Saúde, bem como os sigilos éticos e de privacidade.

Para o cálculo amostral foram analisados os dados de pressão inspiratória máxima (PImáx) e de pressão expiratória máxima (PEmáx) obtidos em um estudo piloto, que incluiu 10 indivíduos. Para este cálculo, comparou-se esses dados com os valores apresentados na literatura e, considerando-se uma diferença a ser detectada de $3 \mathrm{cmH}_{2} \mathrm{O}$, um desvio-padrão de $8 \mathrm{cmH}_{2} \mathrm{O}$, um poder de teste de $95 \%$ com nível de significância de 5\%, estimou-se 28 indivíduos como suficientes para comporem a amostra final.
Foram incluídos no estudo crianças e adolescentes com FC colaborativos, sem doença musculoesquelética, reumática, neurológica, auditiva e visual, e sem doença respiratória aguda no momento da coleta de dados. A estabilidade clínica $^{12}$ foi garantida pela baixa pontuação diante da aplicação dos escores Cystic Fibrosis Clinical Score ${ }^{13}$ e Cystic Fibrosis Fundation Score ${ }^{14}$ no momento da coleta de dados, os quais identificam a presença de exacerbação pulmonar aguda. Também foram registrados dados sobre a colonização, e os genótipos foram consultados por meio da análise dos prontuários; bem como a gravidade da doença classificada pela equipe multidisciplinar segundo o escore de Schwachman Doeurshuk (ESD) ${ }^{15}$. Seriam excluídos os indivíduos que não conseguissem entender a execução do teste ou manifestassem incapacidade em realizar adequadamente a avaliação proposta, o que não ocorreu nesta pesquisa.

Selecionados os participantes, inicialmente realizouse a avaliação antropométrica por meio das medidas de massa (Veta SlimWiso modelo W904i), estatura (estadiômetro fixo da marca Sanny ${ }^{\oplus}$ ) e cálculo de índice de massa corporal (IMC) ${ }^{16}$.

Para avaliação da QV aplicou-se o Cystic Fibrosis Questionnaire, um questionário de qualidade de vida em fibrose cística, (QFC) traduzido e validado para o uso no Brasil por Rozov, Cunha, Nascimento, Quittner, Jardim ${ }^{17}$. Existem quatro versões de QFC: (1) desenvolvidos para crianças (QFC-C) com FC de 6 a 11 anos; (2) para crianças de 12 a 13 anos; (3) indivíduos acima de 14 anos; e (4) para os pais ou responsáveis (QFC-R) dos pacientes de 6 a $13 \operatorname{anos}^{17}$. O questionário foi aplicado conforme a faixa etária, sendo que o mesmo foi aplicado pelo avaliador para as crianças entre 6 e 11 anos. Para os participantes a partir de 12 anos e aos pais/responsáveis pelos pacientes, o questionário foi autoaplicável ${ }^{17}$. As quatro versões dos $\mathrm{QFC}$ abrangem nove domínios de QV, três escalas de sintomas e um item relacionado à percepção de saúde: física, imagem corporal, emocional, social/escola, papel social, vitalidade, alimentação, tratamento digestivo, respiratório, peso e saúde. O escore varia de $0 \mathrm{a}-100$, sendo a maior nota atribuída a um melhor estado de $\mathrm{QV}^{10,17}$.

Por fim, foi realizada a avaliação da FMR por meio da aferição da PImáx e PEmáx com o manovacuômetro digital (MVD300, G-MED ${ }^{\oplus}$, Brasil), respeitando as normas da American Thoracic Society e European Respiratory Society ${ }^{18,19}$. Para se obter a PImáx, a criança foi orientada a expirar até próximo do volume residual 
(VR) e, em seguida, realizar uma inspiração máxima até cerca da capacidade pulmonar total (CPT). A PEmáx foi mensurada a partir de uma inspiração próxima da CPT, seguida de uma expiração máxima até cerca do VR. As medidas foram consideradas aceitáveis (sem vazamento de ar e que foram sustentadas por 2 segundos) e reprodutíveis quando o valor máximo variou menos de $10 \%$ entre si, sendo registrada a maior medida ${ }^{20}$.

Foram realizadas de 3 a 7 manobras para cada uma das medidas de PImáx e PEmáx, e os valores obtidos foram comparados aos propostos nas equações de referência da literatura. Considerou-se a proposta de Rosa et $a .^{20}$ para as crianças de 6 a 10 anos, e as equações de Domènech-Clar et al. ${ }^{21}$ para os pacientes acima de 11 anos.

Todos os dados foram analisados com o software SPSSversion 20.0 for Windows. A distribuição dos dados foi verificada por meio do teste de Shapiro-Wilk e, em seguida, o teste de Wilcoxon foi utilizado para a comparação dos valores de FMR obtidos com os valores preditos na literatura. Os testes de correlação de Spearman (rho) e Pearson (r) foram utilizados para verificar a relação entre as variáveis, considerando a magnitude da correlação como baixa $(r<0,49)$, moderada $(r=0,50$ a 0,69$)$, alta $(r=0,70$ a 0,89$)$ ou excelente $(r>0,90)^{22}$. Uma significância de $5 \%$ foi adotada em toda análise estatística.

\section{RESULTADOS}

Participaram do estudo 28 pacientes, sendo 15 meninos. As características descritivas da amostra estão apresentadas na Tabela 1.

Tabela 1. Caracterização da amostra segundo: idade, massa, altura, IMC, gravidade da doença, parâmetros de PImáx e de PEmáx

$\begin{array}{lr}\text { Variáveis } & \text { Média } \pm \text { DP ou Mediana }\left(\mathbf{I Q}_{25-75}\right) \\ \text { Idade }(\mathrm{anos}) & 10,10 \pm 1,79 \\ \text { Massa }(\mathrm{Kg}) & 29,55(25,60-31,30) \\ \text { Altura }(\mathrm{cm}) & 137,12 \pm 10,74 \\ \text { IMC }\left(\mathrm{Kg} / \mathrm{m}^{2}\right) & 15,84 \pm 1,76 \\ \text { ESD }(\mathrm{pontos}) & 85,00(80,00-90,00) \\ \text { PImáx }\left(\mathrm{CmH}_{2} \mathrm{O}\right) & -57,50(-88,75--45,25) \\ \text { PImáx predita }\left(\mathrm{CmH}_{2} \mathrm{O}\right) & -61,90 \pm 27,46 \\ \text { PEmáx }\left(\mathrm{CmH}_{2} \mathrm{O}\right) & 78,29 \pm 25,30 \\ \text { PEmáx predita }\left(\mathrm{CmH}_{2} \mathrm{O}\right) & 82,50 \pm 24,06\end{array}$

Kg: Quilogramas, cm: centímetros, IMC: Índice de massa corporal, ESD: Escore de Schwachman Doeurshuk, PImáx: Pressão inspiratória máxima, $\mathrm{cmH2O}$ :Centímetros de áqua, PEmáx: Pressão expiratória máxima. Os valores estão apresentados em média $\pm \mathrm{DP}$ para os dados paramétricos, $\mathrm{e}$ mediana $\left(\mathrm{Q}_{25-75}\right)$ para os dados não paramétricos.
A amostra foi composta, em sua maioria, por crianças eutróficas $(71,43 \%)$ e meninos (53,57\%). A colonização apenas por Pseudomonas aeruginosa correspondeu a $28,6 \%$ da amostra, assim como por Staphylococcus aureus. Combinações de 2 ou mais patógenos foram identificadas em $17,8 \%$ dos pacientes e $25 \%$ não apresentaram nenhuma colonização. Quanto ao genótipo, 53,6\% da amostra apresentou mutação $\Delta \mathrm{F} 508$ heterozigoto, 28,6 $\Delta \mathrm{F} 508$ homozigoto, e $17,9 \%$ dos pacientes apresentaram outras mutações.

As variáveis descritivas do QFC-C estão apresentadas na Tabela 2, sendo que este grupo apresentou pontuação compatível com boa QV.

Tabela 2. Pontuações variáveis descritivas do Cystic Fibrosis Questionnaire respondido pelas crianças (QFC-C)

$\begin{array}{lr}\text { Domínios } & \text { Média } \pm \text { DP ou Mediana }\left(\mathbf{I Q}_{25-1}\right) \\ \text { Físico } & 60,18 \pm 24,30 \\ \text { Emocional } & 70,10 \pm 14,40 \\ \text { Social } & 61,90(52,38-71,42) \\ \text { Corpo } & 66,66(44,44-100,00) \\ \text { Alimentação } & 65,50 \pm 25,46 \\ \text { Tratamento } & 88,88(66,66-97,22) \\ \text { Respiratório } & 75,00(50,00-91,66) \\ \text { Digestão } & 100,00(66,66-100,00)\end{array}$

Os valores estão apresentados em média $\pm \mathrm{DP}$ para os dados paramétricos, e mediana $\left(\mid \mathrm{Q}_{25-75}\right)$ para os dados não paramétricos.

As pontuações das variáveis descritivas do QFC-R estão expressas na Tabela 3, sendo observada pontuação compatível com boa QV.

Tabela 3. Pontuações variáveis descritivas do Cystic Fibrosis Questionnaire respondido pelos responsáveis (QFC-R)

\begin{tabular}{|c|c|}
\hline Domínios & Média $\pm D P$ ou Mediana $\left(I Q_{25-75}\right)$ \\
\hline Física & $81,48(68,51-95,55)$ \\
\hline Escolaridade & $77,77(66,66-88,89)$ \\
\hline Vitalidade & $73,33(66,66-80,00)$ \\
\hline Emocional & $86,66(61,66-93,33)$ \\
\hline Corpo & $88,88(66,66-100,00)$ \\
\hline Alimentação & $64,55 \pm 25,32$ \\
\hline Tratamento & $66,66(58,33-88,88)$ \\
\hline Saúde & $77,77(66,66-86,10)$ \\
\hline Peso & $66,66(33,33-66,66)$ \\
\hline Respiratório & $79,25 \pm 17,44$ \\
\hline Digestivo & $72,22(58,33-88,88)$ \\
\hline
\end{tabular}

O teste de Wilcoxon não identificou diferença nos valores obtidos de FMR com o previsto pela literatura, 
tanto para a PImáx $(Z=-0,11 ; \mathrm{p}=0,90)$ como para a PEmáx $(Z=-0,56 ; p=0,56)$.

O resultado das correlações do QFC-C entre a FMR está exposto na Figura 1. Ainda sobre o QFC-C, o IMC apresentou correlação positiva baixa com o domínio alimentação ( $p=0,02 ; r=0,434)$ de acordo com o teste de Pearson. O domínio tratamento apresentou correlação negativa com o genótipo $(\mathrm{p}=0,02 ; \mathrm{rho}=-0,418)$.
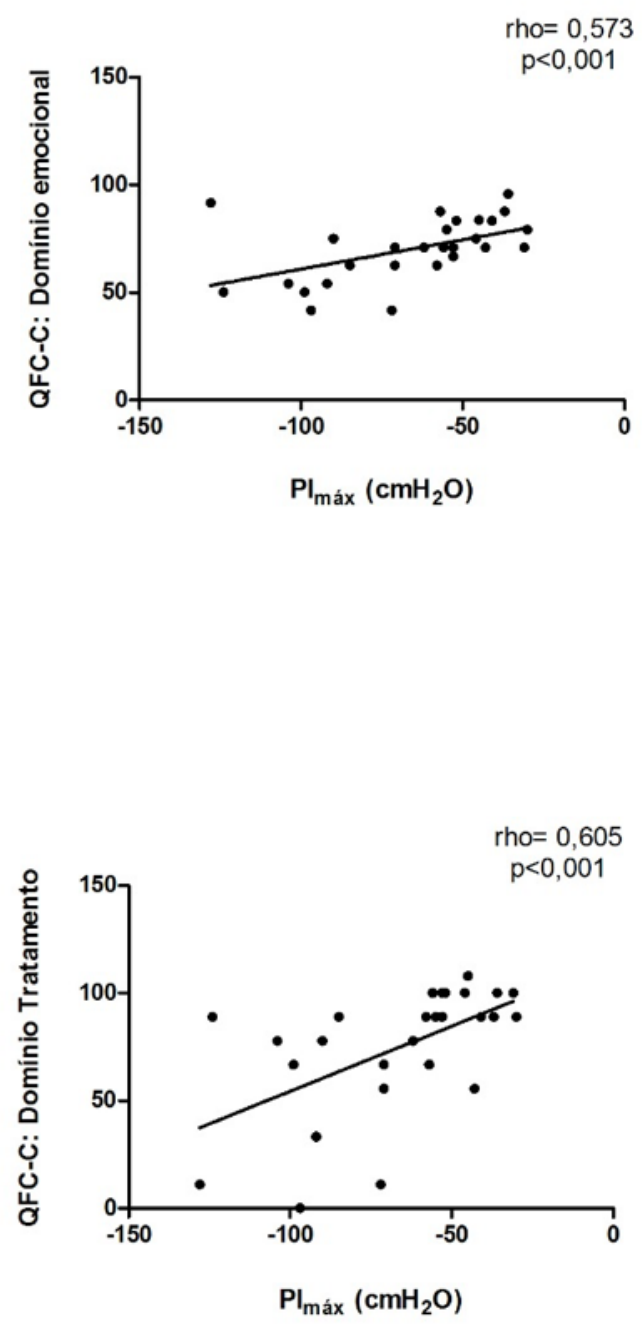

As correlações do QFC-R com a FMR e o ESD estão apresentadas na Figura 2. Além disso verificou-se correlação positiva do IMC entre e os domínios físico $(\mathrm{p}=0,04$; rho=0,387), e peso ( $\mathrm{p}=\leq 0,01 ; \mathrm{rho}=0,588)$ do $\mathrm{QFC}-\mathrm{R}$.

Houve correlação positiva baixa entre o domínio respiratório do QFC-R e o domínio emocional do QFC-C ( $p=0,04$ e $r=0,380)$, de acordo com o teste de Pearson.
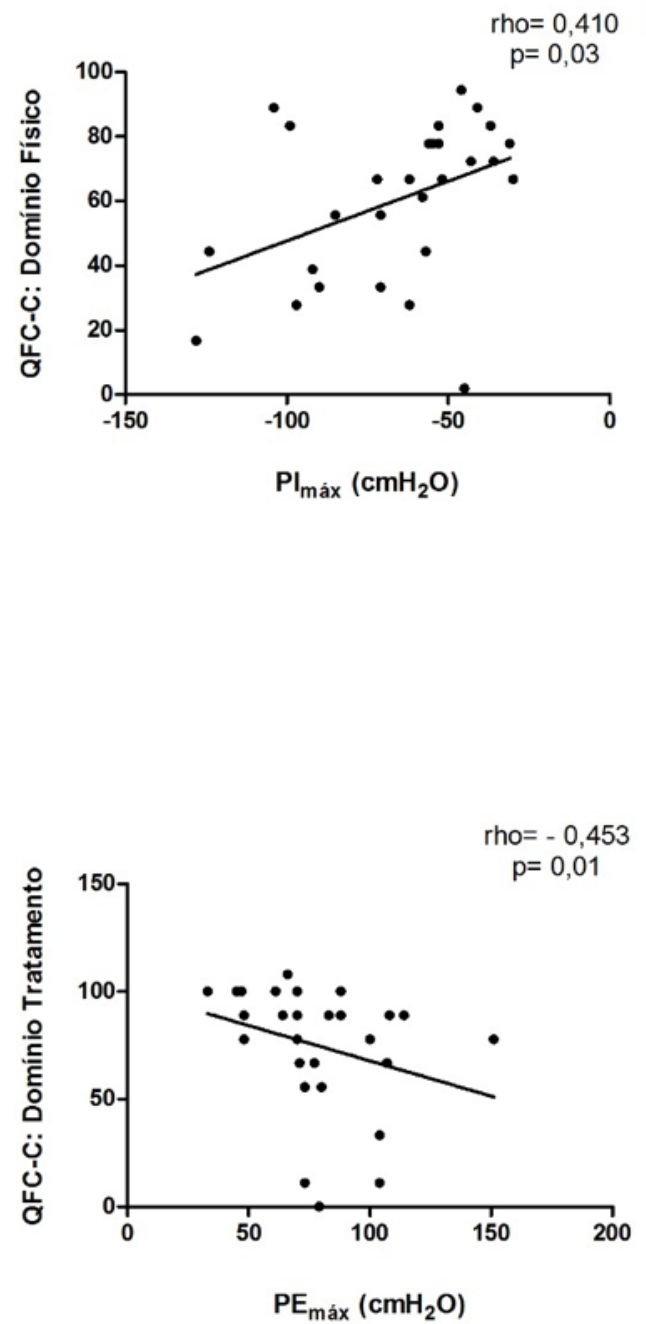

Todas as análises realizadas por teste de correlação de Spearman.

Figura 1. Gráfico de dispersão do QFC-C com parâmetros de PImáx e PEmáx 

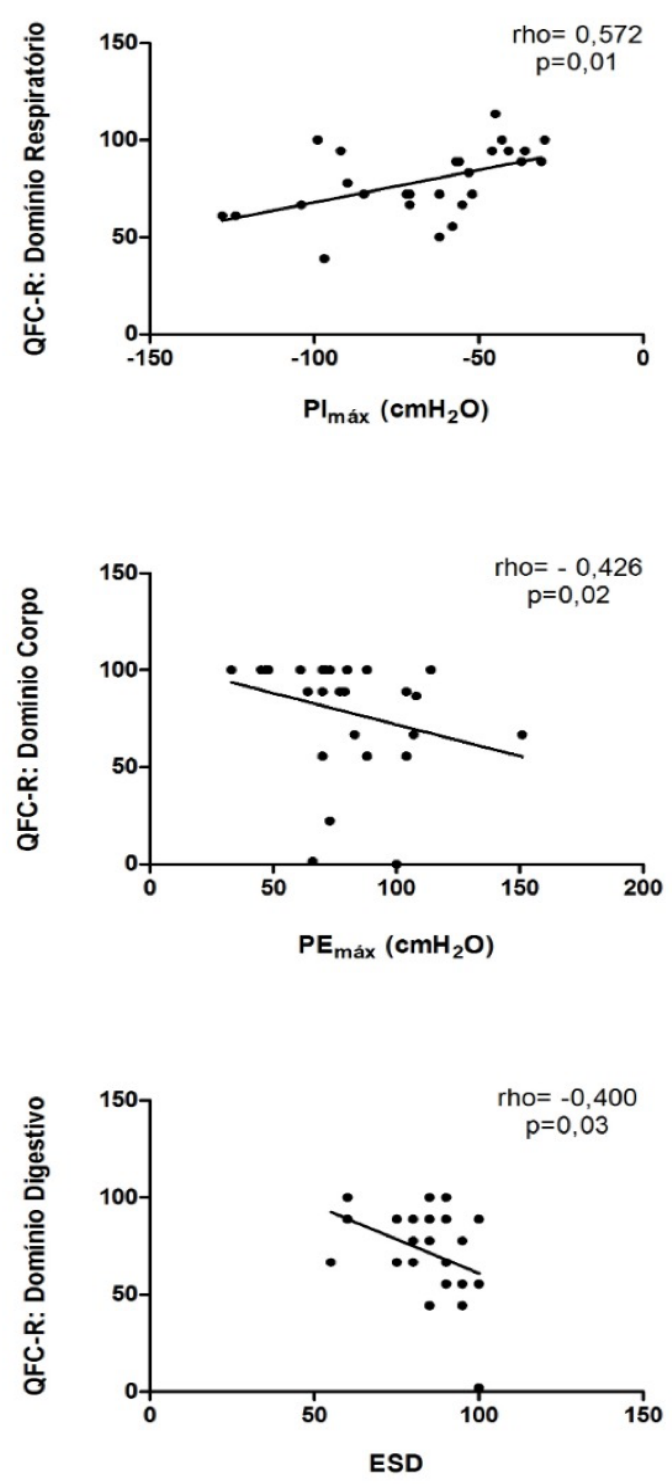

Todas as análises realizadas por teste de correlação de Spearman.

Figura 2. Gráfico de dispersão do QFC-R com parâmetros de PImáx e PEmáx, e com o ESD

\section{DISCUSSÃO}

A amostra estudada avaliou exclusivamente crianças e adolescentes com FC, e apresentou resultados de acordo com a hipótese inicial, na qual o comprometimento da QV associou-se à FMR. Identificou-se que alguns domínios do QFC apontaram correlação positiva com a PImáx, e outros domínios apresentaram correlação negativa com a PEmáx, sendo que os participantes apresentaram uma boa pontuação em relação a QV e valores de FMR próximos aos estabelecidos pela literatura.

Os participantes estavam distribuídos de forma uniforme quanto ao gênero e exibiram boas condições clínicas, com pontuação do ESD classificada como excelente e o IMC dentro ou próximo aos valores de normalidade. A presença de colonização por Pseudomonas aeruginosa foi identificada somente em $28,6 \%$ da amostra. Tais características podem ter influenciado os resultados positivos apresentados, tanto na QV como na FMR. Dados semelhantes foram encontrados no estudo de Vendrusculo et al. ${ }^{9}$, no qual crianças com FC sem colonização por Pseudomonas aeruginosa apresentaram um maior valor da força muscular inspiratória, quando comparadas a crianças hígidas.

Os valores obtidos para PEmáx e PImáx, demonstraramse próximos aos estabelecidos pela literatura ${ }^{14,15}$. O estudo de coorte de Donadio et al. ${ }^{4}$ evidenciou que os pacientes com FC, seguidos por um período de cinco anos, apresentaram leve declínio na função pulmonar e aumento na PImáx, enquanto a capacidade funcional e a PEmáx permaneceram estáveis ${ }^{4}$. Os autores atribuem esses achados ao aumento do trabalho respiratório decorrente da infecção e obstrução das vias aéreas, o que poderia causar um efeito de condicionamento dos músculos respiratórios, refletindo em manutenção ou até aumento da FMR. Essa análise, associada ao fato da amostra exibir boas condições clínicas, pode ser a justificativa dos achados da corrente pesquisa, na qual os pacientes apresentaram valores adequados de PImáx e PEmáx quando comparados ao previsto. No entanto, não houve acompanhamento longitudinal, o que pode ser considerado uma limitação.

Entretanto a literatura aponta resultados divergentes sobre os efeitos que as infecções por patógenos podem apresentar na FMR. Enquanto alguns estudos mostram que a presença de infecção por Pseudomonas aeruginosa propicia um aumento da carga do trabalho respiratório, exercendo, assim, um efeito de condicionamento da $\mathrm{FMR}^{6,23,24}$. Outras pesquisas ${ }^{25,26}$ sugerem que as características da doença crônica, como a desnutrição, infecção recorrente e a hiperinsuflação pulmonar, acabam limitando e diminuindo a FMR.

Em relação a QV, no QFC-R houve uma boa média da pontuação geral para os domínios avaliados, com exceção do domínio peso, resultado este também relatado por Vandeleur et al. ${ }^{27} \mathrm{~A}$ boa pontuação encontrada no QFC-R pode ser reflexo das boas condições clínicas apresentada pelas crianças avaliadas. Este resultado, também pode ser explicado pela percepção e aceitação dos responsáveis em relação à doença e ao físico da criança, como foi relatado no estudo de Santos et al. ${ }^{28}$ Segundo os autores, a família entra em um processo gradativo de aceitação e vivência com a doença crônica, sendo que, muitas vezes, 
não consegue ter uma visão realista da doença e de suas consequências futuras ${ }^{28}$.

Sabe-se que a doença respiratória progressiva acarreta em um aumento da carga imposta nas vias aéreas, podendo comprometer a eficiência e a FMR, o que contribui para a fadiga muscular respiratória e até mesmo com a insuficiência respiratória ${ }^{25}$. O fato de se obter valores apropriados de FMR pode impactar numa melhor competência ventilatória durante as AVD e atividade física ${ }^{8,9}$, o que pode repercutir positivamente nas atividades típicas da infância. Partindo deste pressuposto, a boa pontuação da QV constatada nesta pesquisa, principalmente nos domínios respiratório e corpo, pode indicar o quanto o paciente se sente confortável do ponto de vista respiratório, o que proporciona uma sensação de bem-estar e de saúde. Essa percepção pode permitir que a criança consiga desempenhar melhor suas atividades, o que pode refletir na percepção que os pais têm em relação à saúde da criança.

Foi identificado uma relação inversa entre a pontuação do QV e os valores de PEmáx, de forma que quanto pior a $\mathrm{QV}$, maiores os valores obtidos na manovacuômetria. Esta relação pode retratar a demanda ventilatória destes pacientes ${ }^{6}$. Outros pesquisadores encontraram resultados semelhantes ${ }^{6,9}$ e sugerem que o aumento da FMR se dá pelo maior trabalho respiratório préviamente imposto pela doença, sendo decorrente do quadro de obstrução das vias aéreas.

Observou-se a mesma relação inversa entre a QV e a gravidade de doença detectada pela pontuação no ESD, de forma que, uma maior gravidade da doença pode refletir em uma pior QV. Bodnár et al. ${ }^{29}$ avaliaram 59 pacientes e verificaram uma correlação moderada entre a QV e ESD. Segundo os autores, o ESD pode ser um instrumento útil para fornecer informações, não apenas relacionadas a gravidade clínica da FC, mas também como um indicador de $\mathrm{QV}^{29}$.

Este estudo ressalta uma interessante associação entre os domínios emocional do QFC-C e respiratório do QFC-R, o que pode indicar que o estado emocional do paciente com FC tem influência sobre a condição de saúde deste indivíduo. Recentes estudos ${ }^{25,29,30}$ encontraram alta prevalência de depressão e baixa adesão ao tratamento em populações com FC. Knudsen et al. ${ }^{31}$ identificaram uma frequência de $32,8 \%$ de pacientes com $\mathrm{FC}$ com algum sintoma de depressão, além disso, 74,2\% apresentaram baixa adesão ao tratamento. Sintomas de depressão também estão associados a uma pobre QV e maiores custos em saúde ${ }^{31}$. Desta forma, é relevante a avaliação e o acompanhamento da QV de pacientes com FC e de seus familiares. Esse conhecimento viabiliza a otimização de intervenções terapêuticas, bem como sinaliza aspectos físicos, psíquicos e sociais que devem ser monitorados continuamente nesses pacientes.

Outro importante achado dessa pesquisa foi a presença de correlações positivas da PImáx com a QV, demonstrando que, para a população de crianças estudadas, a força muscular inspiratória adequada tem influência positiva na QV. Estudo recente de Magnet et al. ${ }^{23}$, que avaliou adultos com infecção crônica por Pseudomonas aeruginosa, verificou que a eficiência reduzida da FMR foi associada a redução da QV. Os autores discutem então o fato de, no estágio mais avançado da doença, a FMR apresentar interferência na QV e, neste caso, de uma forma negativa.

Um fator limitante da presente pesquisa se deve à pouca gravidade da doença dos indivíduos avaliados, o que pode ter influenciado nos resultados encontrados. Estudos futuros com populações mais graves devem ser conduzidos para análise da relação entre FMR e QV nessa condição. Além disso, os questionários podem ter sido pouco sensíveis para avaliar a QV destes pacientes, tendo em vista a complexidade das questões e opções de repostas, o que pode dificultar a compreensão do avaliado, principalmente para as crianças de 6 a 13 anos.

\section{CONCLUSÃO}

Esta pesquisa evidenciou que a FMR está associada com a QV de crianças e adolescentes com FC. Os valores da PImáx apontam uma associação positiva com a QV, sugerindo que: quanto maior seus valores, maior a $\mathrm{QV}$ apresentada. Diferentemente, os valores de PEmáx demonstraram uma relação inversa com a QV.Também se identificou relação entre a gravidade da doença com os aspectos nutricionais. Além disso, na amostra estudada os participantes apresentaram valores de FMR acima do esperado e uma boa QV.

\section{REFERÊNCIAS}

1. Reyna SL, Holbrook J, Griffiths HHJ, Peckham D, Mcdermott MF. Dysregulated signalling pathways in innate immune cells with cystic fibrosis mutations. Cell Mol Life Sci. 2020;77:4485-503. doi: 10.1007/s00018-020-03540-9

2. Fiorotto R, Strazzabosco M. Cystic fibrosis-related liver diseases: new paradigm for treatment based on pathophysiology. Clin Liver Dis. 2016;8(5):113-6. doi: 10.1002/cld.583 
3. Cutting GR. Cystic fibrosis genetics: from molecular understanding to clinical application. Nat Rev Genet. 2015;16(1):45-56. doi: 10.1038/nrg3849

4. Grupo brasileiro de estudos de fibrose cística. Registro Brasileiro de Fibrose Cística [Internet]. São Paulo: GBEFC, 2017 [cited 2021 Mar 10]. Available from: http://portalgbefc.org.br/ckfinder/ userfiles/files/REBRAFC_2017.pdf

5. Leroy S, Perez T, Neviere R, Aguilaniu B, Wallaert B. Determinants of dyspnea and alveolar hypoventilation during exercise in cystic fibrosis: impact of inspiratory muscle endurance. J Cyst Fibros. 2011;10(3):159-65. doi: 10.1016/j.jcf.2010.12.006

6. Donadio MVF, Heinzmann-Filho JP, Vendrusculo FM, Frasson PXH, Marostica PJC. Six-Minute walk test results predict risk of hospitalization for youths with cystic fibrosis: a 5-year follow-up study. J Pediatr. 2017;182:204-9. doi: 10.1016/j.jpeds.2016.11.071

7. Saglam M, Vardar-Yagli N, Savci S. Six minute walk test versus incremental shuttle walk test in cystic fibrosis. Pediatr Int. 2016;58(9):887-93. doi: 10.1111/ped.12919

8. Dassios T, Katelari A, Doudounakis S, Dimitriou G. Aerobic exercise and respiratory muscle strength in patients with cystic fibrosis. Respir Med. 2013;107(5):684-90. doi: 10.1016/j. rmed.2013.01.016

9. Vendrusculo FM, Heinzmann-Filho JP, Piva TC, Marostica PJC, Donadio MVF. Inspiratory muscle strength and endurance in children and adolescents with cystic fibrosis. Respir Care. 2016;61(2):184-91. doi: 10.4187/respcare.04231

10. Cohen MA, Ribeiro MÂGO, Ribeiro AF, Ribeiro JD, Morcillo AM. Avaliação da qualidade de vida de pacientes com fibrose cística por meio do Cystic Fibrosis Questionnaire. J Bras Pneumol. 2011;37(2):184-92. doi: 10.1590/S1806-37132011000200008

11. Athanazio RA, Vicente L, Ferreira R, Ribeiro AF, Riedi CA, Procianoy EFA, et al. Diretrizes brasileiras de diagnóstico e tratamento da fibrose cística. J Bras Pneumol. 2017;43(3):219-45. doi: 10.1590/S1806-37562017000000065

12. Santos CIS, Ribeiro JD, Ribeiro AF, Hessel G. Análise crítica dos escores de avaliação de gravidade da fibrose cística: estado da arte. J Bras Pneumol. 2004;30(3):286-98. doi: 10.1590/ S1806-37132004000300016

13. Ramsey B, Boat T. Outcome measures for clinical trials in cystic fibrosis Summary of a Cystic Fibrosis Foundation Consensus Conference. J Pediatr. 1994;124(2):177-92. doi: 10.1016/ S0022-3476(94)70301-9

14. Kanga J, Kuhn R, Craigmyle L, Haverstock D, Church D. Cystic fibrosis clinical score: a new scoring system to evaluate acute pulmonary exacerbation. Clin Ther. 1999;21(8):1343-56. doi: 10.1016/S0149-2918(99)80035-6

15. Doershuk CF, Matthews LRW, Tucker AS, Nudelman H, Eddy G, Wise $M$, et al. A 5 year clinical evaluation of a therapeutic program for patients with cystic fibrosis. J Pediatr. 1964;65(5):677-93. doi: 10.1016/S0022-3476(64)80152-9

16. Biblioteca virtual em saúde. Programa Telessaúde Brasil. Calculadora IMC infantil [Internet]. Brasília (DF): Ministério da Saúde; [modified 2009 Jul 7; cited 2021, Mar 10]. Available from: http://www.telessaudebrasil.org.br/apps/calculadoras

17. Rozov T, Cunha MT, Nascimento O, Quittner AL, Jardim JR. Linguistic validation of cystic fibrosis quality of life questionnaires. J Pediatr. 2006;82(2):151-6. doi: 10.2223/ JPED.1463

18. Gibson GJ, Whitelaw W, Siafakas N. ATS/ERS Statement on respiratory muscle testing. Am J Respir Crit Care Med. 2002;166(4):518-624. doi: 10.1164/rccm.166.4.518

19. Laveneziana P, Albuquerque A, Aliverti A, Babb T, Barreiro E, Dres $M$, et al. ERS statement on respiratory muscle testing at rest and during exercise. Eur Respir J. 2019;53(6):1801214. doi: 10.1183/13993003.01214-2018

20. Rosa GJ, Morcillo AM, de Assumpção MS, Schivinski CIS. Predictive equations for maximal respiratory pressures of children aged 7-10. Brazilian J Phys Ther. 2017;21(1):30-6. doi: 10.1016/j.bjpt.2016.04.002

21. Domènech-Clar R, López-Andreu JA, Compte-Torrero L, DiegoDamiá D, Macián-Gisbert V, Perpiña-Tordera M, et al. Maximal static respiratory pressures in children and adolescents. Pediatr Pulmonol. 2003;35(2):126-32. doi: 10.1002/ppul.10217

22. Munro BH. Statistical methods for health care research. Philadelphia: Lippincott; 2005.

23. Magnet FS, Callegari J, Dieninghoff D, Spielmanns M, Storre JH, Schmoor C, et al. Impact of Pseudomonas aeruginosa infection on respiratory muscle function in adult cystic fibrosis patients. Respiration. 2016;93(1):42-50. doi: 10.1159/000452893

24. Dunnink MA, Doeleman WR, Trappenburg JCA, de Vries WR. Respiratory muscle strength in stable adolescent and adult patients with cystic fibrosis. J Cyst Fibros. 2009;8(1):31-6. doi: 10.1016/j.jcf.2008.07.006

25. Dassios TG, Katelari A, Doudounakis S, Dimitriou G. Chronic Pseudomonas aeruginosa infection and respiratory muscle impairment in cystic fibros. Respir Care. 2014;59(3):363-70. doi: 10.4187/respcare.02549

26. Hahn A, Ankermann T, Claass A, Mann M, Lindemann H, Neubauer BA. Non-invasive tension time index in relation to severity of disease in children with cystic fibrosis. Pediatr Pulmonol. 2008;43(10):973-81. doi: 10.1002/ppul.20887

27. Vandeleur M, Walter LM, Armstrong DS, Robinson P, Nixon GM, Horne RSC. Quality of life and mood in children with cystic fibrosis: associations with sleep quality. J Cyst Fibros. 2018;17(6):811-20. doi: 10.1016/j.jcf.2017.11.021

28. Santos SMR, Duarte TR, Barroso MD, Jesus MCP. Vivências dos familiares frente à criança com fibrose cística. J Heal Sci. 2017;19(2):89-94. doi: 10.17921/2447-8938.2017v19n2p89-94

29. Bodnár R, Kádár L, Szabó L, Hernádi M, Mikóczi M, Mészáros Á. Health related quality of life of children with chronic respiratory conditions. Adv Clin Exp Med. 2015;24(3):487-95. doi: 10.17219/ acem/24991

30. Quittner AL, Goldbeck L, Abbott J, Duff A, Lambrecht P, Solé $A$, et al. Prevalence of depression and anxiety in patients with cystic fibrosis and parent caregivers: results of the international depression epidemiological study across nine countries. Thorax. 2014;69(12):1090-7. doi: 10.1136/thoraxjnl-2014-205983

31. Knudsen KB, Pressler T, Mortensen LH, Jarden M, Skov M, Quittner AL, et al. Associations between adherence, depressive symptoms and health-related quality of life in young adults with cystic fibrosis. Springerplus. 2016;5(1). doi: 10.1186/ s40064-016-2862-5 